\title{
SEMI-BLIND CHANNEL ESTIMATION IN MIMO COMMUMICATION BY TENSOR FACTORIZATION
}

\author{
Bahador Makki Abadi ${ }^{1,2}$, Amir Sarrafzadeh ${ }^{3}$,Foad Ghaderi ${ }^{1}$ and Saeid Sanei ${ }^{1}$ \\ ${ }^{1}$ Centre of Digital Signal Processing, School of Engineering, Cardiff University, Wales, UK \\ ${ }^{2}$ Electrical Engineering Department, Islamic Azad University, Ashtian Branch, Ashtian, IRAN \\ ${ }^{3}$ Department of Communications Engineering, IRAN University of Science and Technology, IRAN
}

\begin{abstract}
In this paper, we introduce a tensor-factorization method for solving channel estimation problem in MIMO applications. we address the estimation problem through a 3-way tensor analysis. We represent the received signals in a $4 \times 4$ MIMO system as a third-order tensor with modes: receiver antennas, packet symbols, and number of packets. Then we demonstrate that the multi-way analysis of PARAFAC2 can be successfully used for solving the MIMO channel estimation problem. This method uses different $\mathrm{m}$-Sequence training symbols for different transmitted signals on the transmitter side. For evaluating the method we applied the well known MMSE-VBLAST detection method to the estimated channel and the results are reported.
\end{abstract}

Index Terms - Tensor Factorization, PARAFAC2, MIMO Detection, Semi-Blind Channel Estimation, m-Sequence, MMSE-VBLAST.

\section{INTRODUCTION}

Multiple-input-multiple-output (MIMO) systems, with multiple antennas employed at both the transmitter and receiver, promise improved performance and bandwidth efficiency compared with conventional systems [1]. MIMO and smart antenna systems are now widely employed to combat the problems of multi-user interference, fading in wireless channels, and to achieve high data rates. Employing entirely pilot data to learn the channel parameters results in poorer spectral efficiency. Moreover, such techniques tend not to use the information in the unknown data symbols to improve channel estimation. Semi-blind techniques can potentially enhance the quality of such estimates by making a more complete use of the available data. With few known training symbols, such techniques can avoid convergence problems associated with blind techniques. Work on semi-blind techniques has been reported earlier for the design of fractional semi-blind equalizers for MIMO channels [2]. Training-based channel estimation methods involve data sequences, which are known both to the transmitter and receiver. Having estimated the channel, the system is capable of decoding the message data to which the training sequence is prefixed. The success of this operation requires the quasi static behavior of the channel [3]. In this paper we used $\mathrm{m}$-sequence training sequences for channel estimation. Parallel factor analysis (PARAFAC) [4] is a well known method for factorizing a multi-way data. Some PARAFAC based MIMO receivers have been developed for code-division multiple access (DS-CDMA) systems [5][6]. In these systems PARAFAC is used to decompose a three-way tensor of the received data (e.g with three modes of spreading diversity-temporal diversity-antenna diversity) for channel estimating and users data detecting. In conventional MIMO applications without using CDMA, the received data is a two-way data with modes of antenna and temporal samples.

Let $S=\left[\mathbf{s}_{1}, \ldots . \mathbf{s}_{n_{T}}\right]^{t}$ denote the transmitted signal where $(.)^{t}$ is transpose operation, then the corresponding received signal vector $X=\left[\mathbf{x}_{1}, \ldots, \mathbf{x}_{n_{R}}\right]^{t}$ is given by

$$
X=H S+E
$$

where, $E=\left[\mathbf{n}_{1}, \ldots, \mathbf{n}_{n_{R}}\right]^{t}$ represents white Gaussian noise matrix observed at the receiving antennas whose rows have zero mean and variance $\sigma_{n}^{2}$. The $n_{R} \times n_{T}$ channel matrix $H$ contains complex Gaussian fading gains. Each entry, $h_{i j}$ is a fading gain between the transmitting antenna $j$ and the receiving antenna $i$. Having quasi-static behavior of channel $H$ we can divide the temporal mode to a limited number of packets passing through a relatively fixed fading channel. With this assumption we have a three-way tensor with (antenna-packet samples-packets) modes and therefore, any tensor factorization scheme can be applied to this data to find the common components of the tensor. PARAFAC2 factorization supports more variability in one mode of the tensor and this results in having more accurate factorization performance rather than that achieved by the traditional PARAFAC method. The reminder of the paper is structured as follows. In Section 2, the tensor factorization methods (PARAFACPARAFAC2) are described. In Section 3, our PARAFAC2 based channel estimation is introduced. In Section 4 the simulations and results are provided. Finally Section 5 concludes the paper. 


\section{PARAFAC AND PARAFAC2}

\subsection{An overview of three-way factorization methods}

PARAFAC [4] model which is used to decompose trilinear data sets with a unique solution, is given below:

$$
X_{i j k}=\sum_{r=1}^{R} H_{i r} F_{j r} C_{k r}+E_{i j k}
$$

where $X_{i j k}$ represents the $i, j, k$-th element in the three-way data set, $R$ is the number of components in common to the three modes, $H_{i r}, F_{j r}$ and $C_{k r}$ are the elements in $H, F$ and $C$ used to obtain the $X_{i j k}$ elements, and $E_{i j k}$ is the residual term. Using tensor notations, the above equation can be presented as:

$$
\begin{aligned}
& X_{(1)}=H(C \odot F)^{t}+E_{(1)} \\
& X_{(2)}=F(C \odot H)^{t}+E_{(2)} \\
& X_{(3)}=C(F \odot H)^{t}+E_{(3)}
\end{aligned}
$$

Where $(.)^{t}$ refers to transpose operation, $\odot$ is Khatri-Rao product and $X_{(n)}$ is unfolded version of tensor $X$ on mode $n$. Using matrix notation, equation (3) can be given as:

$$
X_{k}=F D_{k} H^{t}+E_{k}
$$

for $k=1, \ldots, K$ where $X_{k}$ represents the transposed $k$ th frontal slice of the three-way array $X, H$ and $F$ are the component matrix in the first and second mode, respectively. $D_{k}$ is a diagonal matrix, whose diagonal elements correspond to the $k$ th row of the third component matrix $C$. Finally, $E_{k}$ contains the error terms corresponding to the entries in the $k$ th frontal slice. While direct fitting is applied to the raw data, indirect fitting is applied on covariance matrices of data slices. PARAFAC direct fitting includes an alternative least squares (ALS) optimization method for obtaining $H, F, D_{k}$ for all $k=1, \ldots, K$ and consequently finding three matrices $H, F$ and $C$ of equation (2) respectively [4]. Trilinear ALS fitting method for PARAFAC can be summarized by sequentially estimating $H, F, C$ until convergence.

$$
\begin{aligned}
& H=X_{(1)}\left((C \odot F)^{\dagger}\right)^{\mathrm{t}} \\
& F=X_{(2)}\left((C \odot H)^{\dagger}\right)^{\mathrm{t}} \\
& C=X_{(3)}\left((F \odot H)^{\dagger}\right)^{\mathrm{t}}
\end{aligned}
$$

Where $(.)^{\dagger}$ stands for Moore-Penrose pseudo inverse operation.

\subsection{PARAFAC2}

PARAFAC2 as an extension of PARAFAC is designed to deal with non-trilinear data sets, while keeping uniqueness in the solutions, as the PARAFAC model does. To do so, PARAFAC2 allows a certain freedom in one variable mode. To keep uniqueness in the solutions, all crossproduct matrices $X_{k} X_{k}^{t}$ are forced to be constant over $k$, i.e. $X_{1} X_{1}^{t}=X_{2} X_{2}^{t}=X_{k} X_{k}^{t}$. A similar equation in matrix notation for PARAFAC2 is given as:

$$
X_{k}=F_{k} D_{k} H^{t}+E_{k}
$$

subject to $F_{k}^{t} F_{k}=\Phi, k=1, \ldots K$ where $F_{k}$ is the component matrix in the second mode corresponding to the $k$ th frontal slice, $\Phi$, which is the matrix product of $F_{k}$ and its transpose, is required to be invariant for all slices $k=1, \ldots, K$. In equation (6), we observe that unlike in PARAFAC model, the component matrix in the second mode can vary across slices in PARAFAC2 model. A direct method for fitting PARAFAC2 model is proposed by Kiers [7] and by this method by defining new variables we need to minimize

$$
\sigma\left(P_{1}, \ldots, P_{k}, F, H, D_{1}, \ldots, D_{k}\right)=\sum_{k=1}^{K}\left\|X_{k}-P_{k} F D_{k} H^{t}\right\|^{2}
$$

over all its arguments subject to the constraints $P_{k}^{t} P_{k}=I_{R}$ and $D_{k}$ diagonal, $k=1, \ldots, K$, where $I_{R}$ is an $(R \times R)$ identity matrix. To minimize this function, an ALS algorithm that alternately minimizes equation (7) over $P_{k}$ for fixed $F, D_{k}$ and $H, k=1, \ldots, K$, and minimizes $F, D_{1}, \ldots, D_{K}$ and $H$ for fixed $P_{1}, \ldots, P_{K}$ is used. The algorithm for factorization of a $(I \times J \times K)$ three-way data with $R$ common factors may be summarized into the following steps:

Step 1. Initialize $H$ as the loading matrix from PCA (Principal Component Analysis) on $P_{k} X_{k}^{t} X_{k}$ and initialize $F$ and $D_{1}, \ldots, D_{K}$ as $I_{R}$.

Step 2. Compute the SVD $F D_{k} H^{t} X_{k}^{t}=U_{k} \Delta_{k} V_{k}^{t}$ and update $P_{k}$ as $V_{k} U_{k}^{t}, k=1, \ldots, K$.

Step 3. Update $F, H$ and $D_{1}, \ldots, D_{K}$ by one cycle of a normal PARAFAC algorithm applied to the $(R \times J \times K)$ three-way array with frontal planes $Y_{k}=P_{k}^{t} X_{k}, k=1, \ldots, K$.

Step 4. Evaluate the function value

$\sigma\left(P_{1}, \ldots, P_{K}, F, H, D_{1}, \ldots, D_{K}\right)=\sum_{k=1}^{K}\left\|X_{k}-P_{k} F D_{k} H^{t}\right\|^{2}$. If $\sigma^{\text {old }}-\sigma^{\text {new }}>\epsilon \sigma^{\text {old }}$ for some small value $\epsilon$, repeat Step 2, else exit.

After convergence, there are four matrix outputs as follow: 1 - $H(I \times R)$ related to the first dimension of $X$, in our application it is related to scaled and permuted version of MIMO fading channel.

2- $K P(J \times R)$ related to second dimension of $X$, in our application it is related to temporal samples in each packet. 3- $C(K \times R)$ related to third dimension of $X$ with each row equal to diagonal elements of $D_{k}$.

4- $F(R, R)$ as constant matrix, in our application it is related to covariance matrix of transmitted signals.

Unlike PARAFAC which has only one $P(J, R)$ related to its second dimension, there are $K P(J, R)$. By this variation 
the residual error of PARAFAC2 decomposition is lower than the PARAFAC and better signal or factor reconstruction is achieved for signal separation applications.

\section{PARAFAC2 BASED CHANNEL ESTIMATION}

Regarding PARAFAC2 decomposition and its outputs for each 3-D data $X(I \times J \times K)$ with $R$ factors there are $H \in R_{I \times R}, K$ number of $F \in R_{J \times R}$ and $C \in R_{K \times R}$ as outputs. If this 3-D $X$ data is the output of temporal segmentation for a 2-D mixed data then $I$ will be the number of electrodes which measure the mixed signals, $J$ will be the number of samples in each temporal segments, and $K$ will be number of segments. The outputs of PARAFAC2 decomposing this signal will be $H(I \times R)$ which includes mixing matrix for $R$ sources and the $P_{k} F$ matrices include scaled version of separated samples in each segment and finally $C$ includes scaling factor for each segment. The estimated sources can be calculated from these information. By using PARAFAC2 decomposition, both sources and mixing matrix are achieved simultaneously during each iteration of optimization. Similarly, PARAFAC2 localization method can be used for MIMO channel estimation and also signal detection. In this paper we use a two-way received signal which includes two modes of antenna and temporal samples. We assume that having quasi-static behavior of the channel at a certain time, we can divide the temporal mode to a limited number of packets passing through a relatively fixed fading channel. With this assumption now we have a three-way tensor of $X$ with (antenna, packet symbols, packet) modes. PARAFAC2 blindly estimates channel subject to permutation and also scaling ambiguities. On semi-blind channel estimation method some a priori information about the sources or channel are used to improve the performance of estimation or permutation ambiguity removal technique. We used m-sequence (pseudorandom binary sequence) training sequences as pseudo information attached to the sources to perform our channel estimation.

In this paper in order to estimate the channel we only decompose the received training symbols. After estimating the channel MMSE-VBLAST MIMO detection [8] [9] is used for source separation. The algorithm is more simple than the described PARAFAC2 fitting method because by having known training sequences, we have $F$ as a covariance matrix of training sequeunces, $P_{k}$ is equal to the training sequences in each packet, $D_{k}$ is a diagonal matrix selected as a scaled identity matrix. The only unknown matrix is $H$ or channel which can be simply calculated similar to PARAFAC2 fitting method.

We compute $Y_{k}=P_{k}^{t} \hat{X}_{k}, k=1, \ldots, K$ which $\hat{X}_{k}$ is related to the received training symbols for the $k$ th packet and $P_{k}$ includes the transmitted training symbols. Therefore, instead of applying normal PARAFAC to decompose tensor $Y$ we can have a simple LS optimization method for estimating $H$ [10].
The proposed PARAFAC2 channel estimation can be summarized as follow:

Step1: Compute $Y$ tensor by $Y_{k}=P_{k}^{t} \hat{X}_{k}, k=1, \ldots, K$ which $\hat{X}_{k}$ is the received training symbols for the $k$ th packet and $P_{k}$ includes the transmitted training sequence symbols. Step2: Estimate $H$ from tensor $Y$ by:

$$
\left.H=Y_{(1)}\left((C \odot F)^{\dagger}\right)^{\mathrm{t}}\right]
$$

Where $F(R \times R)$ is the covariance matrix of the training symbols, $C(K \times R)$ is a scaled all one matrix, and finally $Y_{(1)}$ includes all frontal slices of $Y$ tensor.

In this estimation since the only unknown is $H$, The result is not subject to any permutation or sensitive to rank deficiency of the channel. Moreover it is interesting that the overall error of factorization can be used to estimate the input $S N R$. Some detection methods such as MMSE-VBLAST needs noise information as one of their input parameters. So, the second output of our algorithm can be an estimation of $S N R$ on the received signal.

$$
S \hat{N} R=20 \log \left(\left\|H(C \odot F)^{\mathrm{t}}\right\| /\left\|X_{(1)}-H(C \odot F)^{\mathrm{t}}\right\|\right)(9)
$$

MMSE-VBLAST is employed to estimate the sources by using both our estimated channel and the exact channel and compare the results in terms of $B E R$ (Bit Error Rate).

\section{SIMULATED RESULTS}

In this section we evaluated PARAFAC2 channel estimation method for a MIMO system. We selected 4 transmitter and 4 receiver antennas to transfer 4 uncorrelated QPSK baseband symbols between the transmitters and the receivers. We selected two cases of fading channels to evaluate the method. We categorized the channels by varying $r=\lambda_{\max } / \lambda_{\min }$ where $\lambda \mathrm{s}$ are the eigenvalues of fading channel. In the first case we used a full rank uncorrelated channel with $r<10$, and for second case we selected a fading channel with $r>100$. Most of the channel estimation methods are sensitive to channel with high $r$. For both cases we assumed that the fading channel is quasi-static during transmission of the four packets and each packet contains 1000 symbols including $31 \mathrm{~m}$-sequence training symbols and 969 QPSK user data symbols. We calculated the normalized mean square error $N M S E$ for each estimated channel by using

$$
N M S E=(\|H-\hat{H}\|) /\|H\|)^{2}
$$

where ||.|| stands for Frobenius norm. We repeat the measurement of NMSE for different $S N R$ s and 100 times for each $S N R$.

Figure 1 shows the averaged value of $N M S E$ for both cases at different $S N R$ s. It is so interesting that the averaged 
$N M S E$ for estimation of the correlated channels is lower than uncorrelated channels. This means that PARAFAC2 based channel estimation is successful for estimation of both correlated as well as uncorrelated channels.

The results for comparing BERs of MMSE-VBLAST detection method using the estimated and exact channels for the first case are shown in Figure 2. Obviously it can be seen that the difference between the results is considerably low specially at $S N R$ s greater than $10 \mathrm{~dB}$. For the second case having correlated channels, because of higher achieved $B E R$ s it seems that MMSE-VBLAST is sensitive to rank deficiency but in terms of error, for both the estimated channel using PARAFAC2 and the exact channel, the performances are close to each other.

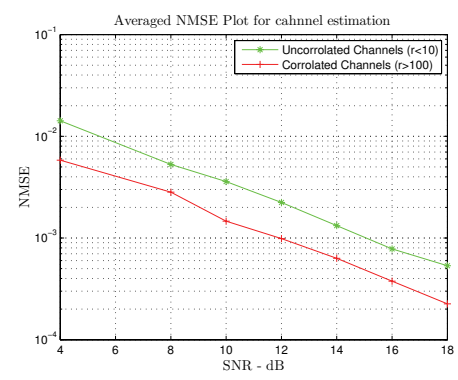

Fig. 1. NMSE Error for both first case(uncorrlated) and second case (corrolated).

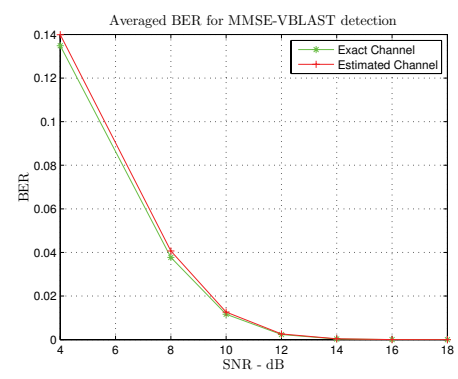

Fig. 2. BER Error for first case $(r<10)$.

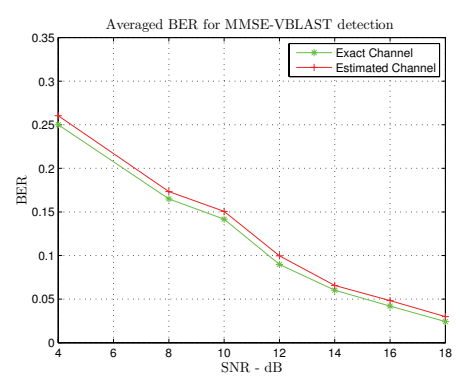

Fig. 3. BER Error for second case $(r>100)$.

\section{CONCLUSION}

In this paper we introduced a tensor factorization method for MIMO channel estimation for two cases of having correlated and uncorrelated channels. We used PARAFAC2 optimization method for estimating the channel using training symbols which are known to both transmitter and receiver. We evaluated the estimated channels using MMSE-VBLAST detection method. The results are promising for uncorrelated case specially for high $S N R$ tests. For correlated channels it seems that the MMSE-VBLAST performance degraded but the performance of our channel estimation method were not deteriorated by rank deficiency of the channel.

\section{REFERENCES}

[1] M. A. Jensen and J. W. Wallace, "A review of antennas and propagation for MIMO wireless communications," Antennas and Propagation, IEEE Transactions on, vol. 52, no. 11, pp. 2810-2824, Nov. 2004.

[2] A. Jagannatham and B.D. Rao, "A semi-blind technique for MIMO channel matrix estimation,” June 2003, pp. 304-308.

[3] K.S. Bialkowski and A. Postula, "Investigations into a semiblind channel estimation technique for 2x2 MIMO system," Antennas and Propagation Society International Symposium, pp. 3257-3260, June 2007.

[4] R. Bro, "PARAFAC. tutorial and applications," Chemometrics and Intelligent Laboratory Systems, vol. 38, no. 2, pp. 149 171, 1997.

[5] N. D. Sidiropoulos, G. B. Giannakis, and R. Bro, "Blind PARAFAC receivers for DS-CDMA systems," Signal Processing, IEEE Transactions on, vol. 48, no. 3, pp. 810-823, March. 2000 .

[6] André L. F. de Almeida, Gérard Favier, and Jo ao C. M. Mota, "Multiuser MIMO system using block space-time spreading and tensor modeling," Signal Processing, vol. 88, no. 10, pp. 2388-2402, 2008.

[7] H. A. L. Kiers, J. M. F. Ten Berge, and R. Bro, "PARAFAC2 - Part I. A direct fitting algorithm for the PARAFAC2 model," Journal of Chemometrics, vol. 13, pp. 275-294, 1999.

[8] J. Benesty, Yiteng Huang, and Jingdong Chen, "A fast recursive algorithm for optimum sequential signal detection in a blast system," Signal Processing, IEEE Transactions on, vol. 51, no. 7, pp. 1722-1730, July 2003.

[9] P. W. Wolniansky, G. J. Foschini, G. D. Golden, and R. A. Valenzuela, "V-blast: an architecture for realizing very high data rates over the rich-scattering wireless channel," Signals, Systems, and Electronics, 1998. ISSSE 98. 1998 URSI International Symposium on, pp. 295-300, 1998.

[10] N. D. Sidiropoulos, R. Bro, and G. B. Giannakis, "Parallel factor analysis in sensor array processing," Signal Processing, IEEE Transactions on, vol. 48, no. 8, pp. 2377-2388, Aug. 2000. 